\title{
SISTEMAS DE GARANTIA DE SEGURANÇA ALIMENTAR APLICADO ÀS EXPLORAÇÕES PECUÁRIAS
}

\author{
Animal health and food safety systems applied to livestock farms \\ Manuel Martins ${ }^{1,2}$, *Ana Cristina Matos ${ }^{1,2,3}$, Ana Claudia Coelho ${ }^{4}$, Telma Brida ${ }^{2}$, Fernando \\ Delgado $^{5}$ \\ ${ }^{1}$ QRural - Unidade de Investigação Qualidade de Vida no Mundo Rural. Instituto Politécnico de \\ Castelo Branco, Portugal, ${ }^{2}$ ESA-IPCB - Escola Superior Agrária, Instituto Politécnico de Castelo \\ Branco, Portugal, ${ }^{3}$ CERNAS - Centro de Recursos Naturais, Ambiente e Sociedade. Instituto \\ Politécnico de Castelo Branco, Portugal, ${ }^{4}$ CECAV - Centro de Ciência Animal e Veterinária, \\ Universidade de Trás-os-Montes e Alto Douro, Portugal, ${ }^{5}$ ESA-IPC - Escola Superior Agrária, \\ Instituto Politécnico de Coimbra, Portugal.
}

*Autor para correspondência: acmatos@ipcb.pt

RESUMO: A segurança dos alimentos deve ser assegurada ao longo do processo de produção, desde a primeira etapa que assenta na produção primária. As questões de segurança alimentar, a globalização, a internacionalização dos mercados e fundamentalmente a preocupação dos consumidores em relação aos riscos alimentares, torna fundamental a adoção de sistemas de garantia de segurança e de qualidade alimentar. Um sistema de garantia de qualidade e de segurança alimentar deve representar uma ferramenta de gestão e de vigilância e deve possuir necessariamente qualidades que o tornem relevantes para o produtor. A legislação na União Europeia obrigou à introdução do sistema HACCP (Hazard Analysis and Critical Control Points) ao longo de toda a cadeia de produção, mas a sua adoção pela produção primária não tem sido consensual. Neste artigo analisam-se as questões associadas à aplicação do sistema HACCP na produção primária com preponderância para a análise de riscos e implicações na saúde animal.

Palavras-chave: alimentos; HACCP; produção primária; qualidade; zoonoses.

ABSTRACT: Food safety must be ensured throughout the production process, from the first stage- the primary production. The questions of food safety, the globalization, the internationalization of markets and mainly the consumer concern about food-related health risks, make it fundamental to implement food safety and quality systems. Food safety and the implementation of quality system must represent a management and monitoring tool and have qualities that make it relevant to the producer. European Union Legislation has forced the introduction of HACCP (Hazard Analysis and Critical Control Points) throughout the entire production chain, but its adoption by primary production has not been consensual. In this work, were analysed the issues associated with the application of the HACCP system in primary production, with preponderance for the risk assessment and implications in animal health.

Keywords: food; HACCP; primary production; quality; zoonosis. 


\section{INTRODUÇÃO}

A segurança dos alimentos deve ser assegurada ao longo do processo de produção, desde a primeira etapa que assenta na produção primária. Assim sendo, a qualidade dos alimentos estará necessariamente associada às atividades desenvolvidas nas explorações pecuárias e agrícolas.

Logo à partida, as doenças zoonóticas com impacto na produção primária e com origem alimentar constituem um risco importante para a produção alimentar porque criam barreiras ao comércio internacional, causam perdas de produtividade, aumentam a morbilidade e a mortalidade humana e animal, e acabam por ter impacto na biodiversidade e no modo de vida dos produtores. Em última instância põem em causa a imagem do sector da produção animal e a confiança dos consumidores, em relação aos produtos de origem animal, realçando as fraquezas dos sistemas de controlo de segurança alimentar.

Durante muitos anos, a promoção da saúde nas explorações assentava principalmente no controlo das doenças zoonóticas, o que acarretava um grande esforço financeiro para a sua erradicação, com programas de ação sanitária focados no efetivo e não no indivíduo.

Posteriormente, o surgimento de várias crises alimentares (Bovine Spongiform Encephalopathy, Listeria, E. coli 0:157 H7, Salmonella Enteritidis, Dioxinas, Foot and Mouth Disease, entre outras) e a crescente evidência científica dos efeitos adversos de certos materiais e substâncias na saúde e no ambiente despoletou uma maior consciência para a necessidade de um desenvolvimento sustentável nos países em desenvolvimento (incluindo a proteção do ambiente) e, também, a consciência da necessidade de melhoria dos métodos de detecção de resíduos químicos. Importa não menorizar o facto de que os perigos acima referenciados poderão amplificar os potenciais riscos para a Saúde Pública face à pressão de estratégias industriais competitivas e do crescimento do comércio internacional.

Ainda que subsista alguma falta de conhecimento científico, já hoje se reconhece a absoluta importância de definir sistemas de certificação que garantam a segurança dos alimentos. Neste campo, a promoção da saúde animal passou a ser feita numa perspectiva integrada e holística, em que, para além dos aspetos inerentes à promoção de saúde, à melhoria da produtividade e à prevenção de doença, são agora objetos de atenção o bemestar animal, a segurança dos alimentos e a sustentabilidade ambiental, para além da saúde ocupacional. Esta abordagem holística enfatiza a perspetiva da cadeia de produção, 'da exploração à mesa do consumidor' (Sossidou et al., 2015; Blaha, 1999; Noordhuizen e Frankena, 1999; Komorowski, 2006; Sorensen et al., 2006), englobando um encadear de questões que por vezes também têm influência na qualidade do produto.

Em simultâneo, o aumento da população mundial com consequente aumento das necessidades proteicas (incluindo a de origem animal), tem vindo a promover alterações dos sistemas de produção, preferenciando uma agricultura intensiva, e - até alterações nas práticas de produção de alimentos para animais.

A par de todas estas modificações da realidade agrícola, observa-se um aumento da mobilidade das pessoas, contribuindo para um aumento da partilha de riscos de doença, situação que resulta exponenciada nos atuais fluxos migratórios.

As alterações climáticas, as alterações dos ecossistemas dos artrópodes vetores, a evolução das populações animais selvagens e as 
suas migrações, o desenvolvimento do turismo (e os fluxos migratórios também), modificaram as características dos riscos na cadeia alimentar. Desde logo, a presença de agentes zoonóticos pode refletir um perfil diferente em função dessas mesmas alterações. Igualmente, o uso na produção animal de substâncias de natureza química como os antimicrobianos e os promotores de crescimento, os aditivos na alimentação animal ou o uso de determinadas moléculas na produção agrícola, como os pesticidas, amplificaram o número de perigos para a saúde pública.

Todas estas alterações levaram a repensar as metodologias de controlo dos alimentos, nomeadamente, os de origem animal, face às novas cadeias de produção e à globalização, tendo em conta também os novos modelos de exposição animal nas explorações e os putativos cenários de doença.

A aprovação, em 1994, dos acordos SPS (Sanitary and Phytosanitary Standards) no âmbito da Organização Mundial do Comércio permitiu liberalizar as trocas comerciais. $E$ ainda que se tenha acautelado os riscos inerentes e os aspetos sanitários de trocas de animais vivos e produtos de origem animal e vegetal, a verdade é que aumentaram os riscos alimentares pela simples multiplicação de vias de infeção e, consequentemente, a preocupação dos consumidores.

Nestes acordos, as medidas requeridas em termos sanitários e fitossanitários devem ser baseadas no conhecimento científico, cujos elementos chave são: análise de risco, regionalização, harmonização, equivalência e transparência (WTO, 1995; Collins e Hall, 2004).

Manter a segurança dos alimentos ao longo das cadeias globais de produção, com mercados cada vez mais globalizados, tornou-se um desafio multifacetado complexo que requer que todos os que produzem, processam, distribuem e vendem produtos alimentares tenham um papel proativo.

Assim, é evidente a necessidade da existência de esquemas de certificação de cada um dos sectores intervenientes na fileira de produção, produção primária, distribuição, processamento e fabrico, embalagem, retalho, serviços (restauração) e preparação em casa pelo consumidor, reforçando a responsabilidades de cada um.

Um sistema de garantia de qualidade e de segurança alimentar deve representar uma ferramenta de gestão e de vigilância e deve possuir necessariamente qualidades que 0 tornem relevantes para o produtor. Deve ser operacional, eficiente e economicamente benéfico (Sorensen et al., 2004).

Neste trabalho analisam-se as questões associadas à aplicação do sistema HACCP na produção primária com preponderância para os riscos e implicações na saúde animal.

\section{DESENVOLVIMENTO}

\section{O HACCP e as explorações pecuárias}

O HACCP (incluído no Codex Alimentarius em 1989 e que tem vindo a ser incorporado na ISO 22000) centrase na eliminação ou no controlo dos fatores de risco associados com 0 processo de produção, em função da definição de pontos críticos (PC), indicadores de um aumento de um risco associado a um perigo. Ele introduz medidas corretivas pré-definidas quando é ultrapassado um valor crítico (risco aceitável). Pode ser implementado pelo produtor, reconhecendo-lhe um papel chave na garantia de colocação de alimentos seguros no mercado, tendo o seu processo de produção como ponto de partida.

Ao nível da União Europeia, a Diretiva 93/43/CEE do Conselho, de 14 
de Junho de 1993, reconheceu o sistema HACCP, considerado como um sistema de gestão de risco (Coleman e Marks, 1999) baseado na análise de cada passo da produção de um alimento, com identificação de potenciais perigos e de pontos de controlo ao nível de toda a cadeia alimentar.

Posteriormente, foi definido o chamado "pacote de higiene" relativo à higiene dos géneros alimentícios (Regulamentos (CE) 852, 853, 854/2004) e legislação referente aos princípios gerais que regem os géneros alimentícios (Regulamento (CE) 178/2002) que levaram à obrigatoriedade da implementação do sistema HACCP (Regulamento (CE) 852/2004) em todos os processos e produção alimentar, a partir 1 de janeiro de 2006.

O sistema HACCP assegura que o produto final atinja especificações que determinam a sua segurança e a proteção do consumidor, tendo sido aplicado em vários sectores da indústria alimentar com sucesso, mas não de forma homogénea, sobretudo devido a algumas barreiras técnicas (Panisello e Quantick, 2001).

No entanto, apesar de todos os regulamentos, o sistema HACCP não foi tornado obrigatório para a produção primária. Em lugar de caracter obrigatório, houve apenas uma indicação preferencial para a sua aplicação, devendo respeitar os requerimentos específicos do Anexo I do Regulamento (CE) 852/2004 e do Regulamento (CE) 853/2004.

Sendo 0 HACCP altamente específico e, tal como se verifica nos outros sectores da cadeia alimentar, também aqui, no tecido primário, deve ser aplicado individualmente a cada da exploração (Lievaart et al., 2005). sistema tem vindo a ser aplicado a vários tipos de produção, especialmente em explorações de bovinos de leite
(Ruegg, 2002; Sorensen et al. 2004; Silva e Noordhuizen, 2008; Boersema et al., 2008; Noordhuizen e Silva, 2009; Vilar et al., 2012), mas também em explorações de bovinos de carne (Adam e Brulisauer, 2010), em explorações de suínos (Noordhuizen e Frankena, 1999; Bonde e Sorensen, 2004; Horchner e Pointon, 2011; Beekhuis-Gibbon et al., 2011a; Beekhuis-Gibbon et al., 2011b; Van der Spiegel et al., 2013), em explorações de caprinos (Nettles e Bukenya, 2005; Malher e Noordhuizen, 2008), e nas explorações de aves (Kaitazov e Stoyanchev, 2008), de animais exóticos (Moller, 2004), e na apicultura (Formato et al., 2011; Formato e Smulders, 2011).

A aplicação do HACCP às explorações pecuárias não é, no entanto, unânime (Keener, 2001). Noordhuizen e Welpelo (1996) referemse à sua especificidade nas explorações, considerando-o como um sistema que implica um menor aporte de trabalho, com necessidade de pouca documentação, focado na gestão operacional e tático (bem planeado), e relevam como fatores positivos o facto das medidas e do estatuto sanitário dos rebanhos serem demonstráveis aliados à possibilidade de haver ligações com outros componentes da cadeia de segurança de qualidade alimentar.

Sperber (2005) refere que o HACCP criou falsas expectativas devido à falta de medidas de controlo efetivas neste ponto da cadeia alimentar. No entanto este autor reporta que raramente as falhas de segurança nos alimentos, se devem a falhas no HACCP, mas sim ao nível dos prérequisitos.

No entanto, Cullor (1997) referiu, por exemplo, ser difícil melhorar substancialmente a segurança dos alimentos utilizando o HACCP nas explorações, alegando que não há nenhuma evidência científica que suporte qualquer PC disponível na 
exploração para a implementação da redução ou eliminação de $E$. coli 0157:H7, ou seja, sempre que não se reúnam as principais características necessárias à erradicação do agente (ou seja, um hospedeiro único, sem reservatório selvagem, produção de sinais e sintomas identificáveis de doença clínica, e disponibilidade de um diagnóstico eficiente).

Alguns autores preconizam $\mathrm{O}$ desenvolvimento de planos de HACCP genéricos para o sector que podem diferenciar claramente entre prérequisitos e o próprio plano HACCP. Após o desenvolvimento destes planos genéricos, cada produtor pode ajustálos à sua situação com o auxílio de especialistas. Os planos de erradicação de doença, nomeadamente de zoonoses e a sua certificação, os planos de biossegurança, o controlo de doença não infeciosa são componentes do processo, podendo funcionar como prérequisitos ao HACCP. Outras abordagens são referenciadas e complementares aos planos de HACCP, como os sistemas de gestão da produção e de saúde de rebanho e a avaliação SWOT (Strenght; Weakness; Opportunities and Threats) (Noordhuizen e Silva, 2007).

Nas explorações pecuárias, os próprios animais e o ambiente envolvente são as fontes primárias de muitos dos perigos associados aos alimentos, sobretudo perigos de natureza microbiológica e química, e, consequentemente, os sistemas de produção animal apresentarão, assim, vários riscos relacionados com a natureza dos processos de produção, muitos tão complexos que têm tornado difícil a implementação do HACCP na produção primária.

Por isso, têm sido desenvolvidos vários sistemas de certificação para as explorações (Baines et al., 2004) dos quais se salienta o GlobalGap, para além dos requerimentos de segurança dos alimentos standard impostos pelas cadeias de distribuição, que negoceiam diretamente com os produtores, em relação a alguns produtos de origem animal.

\section{Análise de risco}

Associado ao sistema HACCP, deve ser feita uma análise de risco seguindo o Regulamento (CE) $178 / 2002$, que não só estabelece as diretrizes principais de segurança de alimentos e de alimentos para animais, como define um nível de risco compatível, isto é, um limite 'seguro' (aceitável/tolerável) em relação a cada potencial perigo (Romero-Barrios et al., 2013).

No entanto, é extremamente difícil reportar $o$ número de atividades desenvolvidas numa exploração e os perigos associados. Por isso é necessário caracterizar cada exploração face à variabilidade das mesmas quer em termos estruturais e organizativos, quer em termos de modo produção e de objetivos, o que dificulta a análise de risco.

A análise de risco é normalmente aceite para definir o impacto de um perigo ou o nível apropriado de proteção da saúde pública e o estabelecimento de linhas gerais a respeitar em termos de segurança de alimentos (Gorris, 2002).

Ela permite identificar fatores de risco (específicos para cada exploração) e determinar os PC em função de níveis de risco aceitáveis, para ajudar a definir esquemas de monitorização e para selecionar medidas preventivas (gestão de risco), visando a manutenção da saúde animal, associada a uma análise de custo benefício (deve haver um mínimo de PC compatíveis com os recursos humanos e financeiros disponíveis).

A avaliação de risco tem como objetivo identificar e quantificar a magnitude do risco, face aos diferentes níveis de risco. Esta avaliação permite 
ao gestor de risco optar pelas medidas mais adequadas, interrelacionando as etapas de produção na exploração, numa perspetiva holística e tendo em atenção os pré-requisitos implementados e outros aspetos que não são objeto de atenção dos avaliadores de risco.

\section{Embora}

possam

ocorrer

contaminações cruzadas entre as diferentes etapas, estas não são consideradas pela avaliação de risco realizada etapa a etapa, face à diversidade de etapas e no modo como se interrelacionam que se observam em diferentes explorações, o que deve ser tido em atenção quando se usam tabelas de avaliação de risco.

No entanto, é possível que seja necessário introduzir algum ajustamento dos pré-requisitos já instituídos, após a avaliação de risco. É importante ter em conta as necessidades e preocupação do consumidor, para melhor dirigir as medidas de gestão e a melhoria da saúde e do bem-estar animal, não esquecendo as preocupações do impacto ambiental.

A avaliação refere-se a probabilidades e efeitos (Lievaart et al., 2005), pelo que para cada etapa do sistema de produção é necessário listar os potenciais perigos associados, considerando a interação entre a probabilidade de ocorrência (poderá ser a prevalência ou a incidência do perigo) e o seu impacto (saúde pública e económico) para quantificar o risco, sendo importante objetivar a ação dos perigos definidos em função do processo de produção (morbilidade, mortalidade, letalidade, produtividade, reprodução, entre outros). Para um perigo ser considerado significativo deve ser razoavelmente provável a sua ocorrência e as consequências devem ser relativamente importantes.

A avaliação de risco deve envolver necessariamente um número considerável de especialistas face às diferentes condições e situações nas explorações. De acordo com a OIE (World Organisation for Animal Health) (Vose, 2008) é necessário ter conhecimento sobre introdução, emissão e transmissão do agente em causa, que deve ser realizado por especialistas e deve ter em conta a legislação.

Cada produtor produz para um determinado segmento de mercado, tendo por isso problemas de saúde específicos, associados a fatores de risco em condições ambientais próprias de cada exploração. O conhecimento sobre estes fatores de risco são igualmente fundamentais para a definição de programas de biossegurança e outros são objeto de um código de boas práticas.

Devem ainda ser consideradas as metodologias a adotar em termos de diagnóstico, nomeadamente em relação aos seus valores intrínsecos e extrínsecos, assim como os seus limiares de deteção.

\section{Pré-requisitos ao HACCP}

Antes da implementação do HACCP é importante a adoção de prérequisitos ao sistema HACCP (Alexoupoulos et al., 2004), que constituem procedimentos definidos para assegurar uma produção alimentar e um manuseamento ambiental favorável à produção de alimentos seguros.

Estes requisitos são importantes para o controlo de riscos associados à produção animal, nomeadamente em relação à rastreabilidade, à identificação dos perigos, aos registos, às deslocações, aos alimentos, à água, às instalações pecuárias, à higienização e aos produtos químicos utilizados, à saúde animal, à biossegurança, aos planos de desparasitação e de vacinação, aos medicamentos veterinários, aos fitossanitários, à qualidade do produto, à eliminação do estrume e do chorume, à maquinaria e 
equipamentos diversos, à presença de animais de companhia e selvagens, e outros mais específicos associados a cada processo de produção e que podem comprometer a segurança e a qualidade do produto final.

Os códigos de boas práticas agrícolas e de higiene constantes do Anexo I do Regulamento (CE) 852/2004 fazem referência a diversas medidas de redução ou prevenção de riscos gerais, mas a sua adoção não constitui por si só garantia de uma efetiva eliminação dos perigos considerados pela Saúde Pública (Copa - Cogeca, 2015; Maunsell e Bolton, 2004; WOAH, 2006).

Implementam-se procedimentos operacionais padronizados que garantam uma qualidade desejável dos produtos, em cada passo da sua produção, permitindo alcançar os objetivos definidos pelas boas práticas de produção, eliminando desde logo a probabilidade de ocorrência de muitos dos perigos que ameaçam a qualidade e segurança dos produtos em causa.

Os operadores da produção primária devem cumprir a parte $A$ do Anexo I do Regulamento (CE) 852/2004 onde estão descritas as medidas gerais de higiene (controlo da contaminação; ambiente, saúde animal e bem-estar que possam implicar a saúde humana) e o Regulamento (CE) 183/2005, em relação a alimentos para animais, ambos contribuindo para elevar o nível de segurança dos alimentos na União Europeia.

\section{A implementação do HACCP}

A correta implementação do sistema HACCP requer que passos cientificamente documentados e medidas preventivas, possam ser efetivamente aplicados nos pontos críticos.

O HACCP consiste em 7 princípios (que requerem 12 passos para a sua implementação) divididos em 3 atividades de gestão (Codex Alimentarius Commission, 1997; Codex
Alimentarius Comission 1998) (Figura 1).

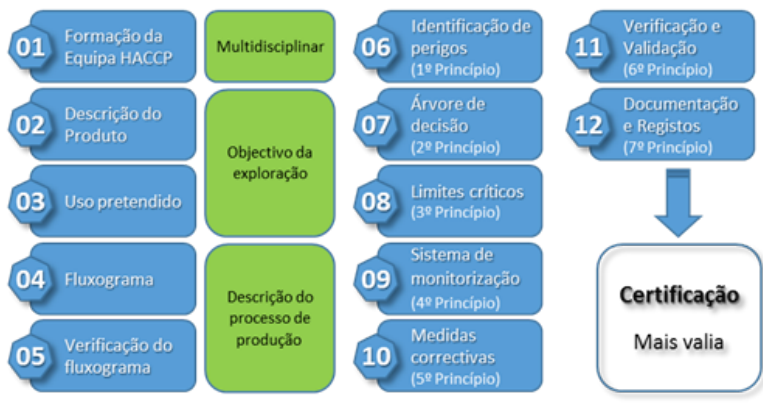

Figura 1 - Implementação do HACCP (7 princípios - 12 passos).

\section{Passo 1 - Equipa HACCP}

Este passo assenta na definição de uma equipa HACCP e na identificação das pessoas envolvidas (produtor e funcionários, especialistas externos, como por exemplo o médico veterinário assistente, autoridades sanitárias, agrónomos, nutricionistas, técnicos de manutenção de equipamentos, entre outros).

É importante o envolvimento de todos os interessados, nomeadamente aqueles ligados à saúde pública e saúde animal, no desenvolvimento de programas de emergência de resposta à ocorrência de surtos de doença (troca de informação acerca dos riscos associados aos produtos alimentares, protocolos, e medidas a tomar tendo em conta os riscos considerados).

\section{Passo 2 - Produto}

Inclui a descrição do produto e a sua distribuição, intimamente interrelacionado com o objetivo de produção.

Deve ser feita uma descrição pormenorizada da exploração e da estrutura de produção, que assume formas específicas de organização, sendo fundamental avaliar os indicadores objetivos de produtividade e do foro reprodutivo, indicadores indiretos de organização do efetivo, e indicadores económicos, que caso não existam, deve ser considerada a sua criação. 
A equipa deve: [1] definir o caminho a seguir em função do objetivo de produção; [2] caracterizar a exploração e o processo de produção, tendo em conta a localização, o layout da exploração, o clima, a rede de acessibilidades, os cursos de água, as explorações vizinhas, as zonas vedadas (cercas, muros), as instalações, a maquinaria, os parques, as pastagens, o local de entrada e de acesso de veículos, os técnicos e visitantes, os inputs de produtos e serviços; [3] desenvolver fluxogramas, e [4] definir os perigos a considerar e as ações a desenvolver.

Torna-se indispensável estabelecer um perfil de saúde da exploração definido, em última instância, pela demografia populacional e pelos valores de produção (Rosenberg, 1986). A saúde animal deverá ser entendida não só como a presença ou ausência de uma doença de etiologia específica, mas também como um conjunto de condições que determinam as características de produção de uma população animal num determinado tempo e local.

\section{Passo 3 - Utilização do produto}

Neste $3^{\circ}$ passo procede-se à Identificação do uso pretendido. É preciso identificar os produtos produzidos na exploração, incluindo as suas propriedades e a intenção da sua utilização, nomeadamente se o produto será para venda local, como acontece por exemplo com os queijos de ovelha e cabra produzidos a partir de leite cru.

\section{Passo 4 - Fluxograma}

A construção de um fluxograma que resulta num documento, onde se faz a descrição de todos os passos do processo de produção animal e que vai funcionar como ferramenta de comunicação entre as pessoas envolvidas, com as especificações bem representadas; não há uma exploração tipo e as condições particulares estão normalmente na origem de problemas de segurança.

Este fluxograma pode ser geral, mas assente numa base diária e alguns passos do processo devem ser detalhados para permitir uma melhor compreensão sobre quais os perigos a considerar e onde, para identificar a transferência dos diferentes perigos entre etapas e atividades (Cerf e Donnat, 2011), facilitando a avaliação do risco num determinado passo do processo de produção e as condições que estão interrelacionadas (Lievaart et al., 2005), assim como, a identificação de pontos críticos e quais as medidas preventivas ou corretivas que podem ser tomadas.

Assim, para a definição de um processo de produção de qualidade é fundamental que as diferentes áreas na exploração possam estar identificadas e posteriormente, em cada uma das áreas, sejam identificados os potenciais perigos assim como as condições de risco associado (Noordhuizen e Silva, 2009).

$\mathrm{Na}$ sua construção pode ser utilizado o mapa da exploração (Maunsell e Bolton, 2004; Malher e Noordhuizen, 2008), podendo recorrerse ao cadastro da exploração e aos ortofotomapas (Sistema de Identificação de Parcelas, Regulamentos (UE) 1306 e $1307 / 2013$ e 640 e 907/2014).

\section{Passo 5 - Verificação do fluxograma}

Verificação do fluxograma no local de forma a permitir ajustamentos. Estes dois passos, 4 e 5, são críticos porque devem descrever o processo de produção, de uma forma precisa e cuidadosa.

\section{Passo 6 (Princípio 1)}

Passo referente à identificação de perigos, que também é uma etapa da análise de risco, considerada de forma independente pela OIE (Vose, 2002) ou associada à avaliação de risco (Codex 
Alimentarius Comission, 1997; Murray et al., 2004a; Murray et al., 2004b).

É importante priorizar os perigos com origem alimentar mais relevantes, que ameaçam diariamente a saúde dos consumidores e neste contexto, qualquer política eficaz por parte das agências de segurança da cadeia alimentar deve concentrar-se nos mais pertinentes (Cardoen et al., 2009), tendo em conta a sua prevalência (nem sempre documentada).

Importa fazer ênfase ao facto de que esta priorização e a sua hierarquização constituem uma ferramenta importante de decisão, em matéria de saúde animal, para selecionar os riscos, ligados à doença, que devam ser avaliados pelas políticas de saúde pública (Humblet et al., 2012).

Para além das doenças incluídas nos planos de erradicação, o plano deve também abranger os agentes que 0 produtor entenda querer evitar que entrem na exploração (biossegurança) (FAO, 2010; OIE, 2010) e que não tenham necessariamente impacto na saúde pública, assim como aqueles cujo impacto esteja associado à qualidade do produto.

Em 1971, a $2^{a}$ edição do Código de Saúde Animal Terreste publicado pela OIE, de entre 43 doenças referenciadas, apenas 3 eram consideradas como zoonoses de origem alimentar (brucelose, tuberculose e triquinelose), subindo nos anos seguintes até 13 entre 30 doenças classificadas como zoonoses em 2012, representando uma preocupação para os sectores industriais agro-alimentares (Berman e Shimshony, 2013). Mais de $70 \%$ das doenças humanas têm um reservatório animal (Brugère- Picoux e Kodjo, 2007).

Cerca de $10 \%$ da população de países industrializados sofre de uma doença de origem alimentar. Por sua vez, nos países em desenvolvimento, cerca de 2,2 milhões de pessoas morrem com gastroenterite, numa grande proporção com origem alimentar. Tais factos tornam necessária uma intervenção rápida para evitar a ocorrência destes eventos, introduzindo medidas que possam prevenir ou reduzir o risco de doença alimentar, aplicadas a toda a fileira alimentar, incluindo a produção primária (Maunsell e Bolton, 2004; WHO, 2008). Três em cada quatro agentes patogénicos emergentes que afetam o homem têm origem nos animais ou nos seus produtos (Taylor et al., 2001).

Assim, nas últimas duas décadas as crises sanitárias multiplicaram-se e novas patologias apareceram e desapareceram (fenómenos de emergência e reemergência), de causas múltiplas e com relações de causaefeito muitas vezes difíceis de compreender, tais como o Vírus de Nipah na Ásia (1999), Febre Aftosa na Europa (2001), Gripe Aviária por todo o mundo (2004), Síndrome Respiratória Aguda Severa (SRAS) (2002), e outros como Ébola, Febre do Vale do Rift, Febre do Nilo Ocidental, Febre Catarral Ovina (língua azul), com maior ou menor ligação aos alimentos de origem animal, mas que aumentam a desconfiança entre os consumidores.

Os perigos biológicos envolvem agentes patogénicos como bactérias (Salmonella spp., Yersinia enterocolitica, Brucella sp., Mycobacterium bovis, Campylobacter jejuni, Listeria monocytogenes, Escherichia coli, Bacillus cereus, Staphylococcus aureus, Clostridium botulinum, entre outras), vírus, parasitas (Cryptosporidium parvum, Giardia spp., Trichinella spp.) e fungos (Aspergilus spp., Fusarium spp.).

O Homem contamina-se pelo contacto direto com os animais ou pelo consumo de produtos alimentares com agentes patogénicos. Alguns destes agentes são objeto de programas de erradicação cofinanciados, como é o 
caso da Brucella melitensis nos pequenos ruminantes.

Os principais perigos biológicos a considerar não passam exclusivamente pelos agentes que provocam surtos de doença, envolvendo em muitos casos um alimento específico, mas muitas vezes centram-se em casos esporádicos, cuja fonte não é identificada.

Por outro lado, muitos dos agentes patogénicos presentes nos alimentos são de origem fecal e a contaminação dos produtos acontece num período precoce da sua produção. Muitos destes perigos estão associados à carne, ao leite e aos seus derivados, mas muitas vezes não implicam a doença clínica, nem perdas de produção ou outros custos diretamente tangíveis para os produtores (Gardner, 1997).

Muitas vezes, os animais são portadores assintomáticos, podendo eliminar os agentes de forma intermitente, dificultando a sua deteção. Muitos são eliminados pelas fezes, contaminando alimentos e água e originando doenças de curta duração e com sintomatologia leve. Outros agentes, como Listeria monocytogenes, podem ocorrer tanto nas explorações como na indústria (Van der Spiegel et al., 2013).

Para além dos agentes infeciosos, é necessário ainda considerar os perigos químicos: resíduos de óleo do equipamento, detergentes e desinfetantes (nestes deve-se considerar a eficácia face à presença de matéria orgânica), pesticidas, herbicidas, medicamentos veterinários (antibióticos, antiparasitários, hormonas, entre outros), dioxinas, metais pesados, elementos radioativos e PCB's (policlorobifenilos). Alguns causam efeitos a longo prazo, muitas vezes de difícil ligação com a ocorrência de doença, mas com impacto na saúde animal e na saúde pública, levando a uma reformulação do conceito de zoonose (Frazzoli e Mantovani, 2009). Muitos destes perigos só poderão ser reduzidos e/ou eliminados na própria exploração.

Em relação aos alimentos para animais, o seu controlo está dependente dos programas adequados de controlo de resíduos químicos, ao nível da produção destes alimentos, antes da sua entrega nas explorações. Os produtores devem assegurar garantias de segurança em relação aos agentes químicos já referenciados, com especial atenção para as micotoxinas, e em relação aos produtores que compram aditivos e pré-misturas, devem cumprir o Anexo II do Regulamento (CE) 183/2005.

De entre os perigos físicos podese considerar: pedras, solo, metal, vidro, plásticos, cornos, agulhas, maioritariamente controlados pelos prérequisitos ao HACCP, nomeadamente pela aplicação de boas práticas.

Malher e Nordhuizen (2008) consideram ainda os perigos de maneio que se relacionam por exemplo com problemas metabólicos como a acidose, com índices de crescimento ou de performance reprodutiva.

Após a identificação dos perigos mais relevantes na exploração, há que definir as condições de risco associadas à sua presença. Devem, por isso, ser formulados requerimentos específicos (objetivos e critérios de performance, tendo em conta os Objetivos de Segurança Alimentar) para a definição de um sistema de garantia de segurança baseado no risco, e os produtores devem garantir que os seus produtos respeitam esses requerimentos específicos (Snijders e Van Knapen, 2002; Meyer-Broseta e Evain, 2004).

Passos 7, 8 e 9 (Princípios 2, 3 e 4)

Estes 3 passos - Passo 7 (Princípio 2 - Aplicação da árvore de decisão do HACCP), Passo 8 (Princípio 3 - Estabelecer os valores alvo e de 
tolerância para assegurar que o PC está sob controlo) e Passo 9 (Princípio 4 estabelecer o sistema de monitorização para assegurar o controlo do PC, assim como o responsável por essa monitorização) merecem uma abordagem conjunta.

Sorensen et al. (2004) referem que a metodologia HACCP precisa de responder a uma série de questões relacionadas com a qualidade e não ficar confinada a riscos relacionados com a saúde pública. O objetivo é a prevenção de problemas específicos utilizando um controlo contínuo em pontos críticos, que indicam um aumento de risco de certos problemas. Se um valor de alarme para um certo ponto crítico é atingido, então deve ser tomada uma ação predefinida de forma a diminuir o risco.

Estes passos pretendem identificar os pontos, os procedimentos e os momentos operacionais (qualquer estado no processo de produção) onde será possível controlar os perigos associados aos alimentos, no sentido de os prevenir, eliminar ou minimizar a probabilidade de ocorrência ou reduzir a um nível considerado como aceitável.

Os PC são o elemento-chave do plano HACCP (Lievaart et al., 2005). Referem-se aos perigos considerados importantes no processo de produção e no produto final, não controlados pelos pré-requisitos, devendo ser mensuráveis no processo de produção e determinar uma medida de controlo exequível em função de critérios de performance desejados, associada com padrões e níveis de tolerância (objetivos de performance).

O controlo deve ser dirigido para a eliminação ou redução do risco. Contudo, nem sempre se podem associar a valores de referência sobretudo para os perigos biológicos ou de maneio (por ex. compra de animais) (Lievaart et al., 2005).
Assim, para que um ponto possa ser considerado crítico é necessário que possa estar associado a um determinado perigo, que esteja estabelecido um limite crítico (critério que divide a aceitabilidade da não aceitabilidade) e ainda que seja realizada a sua validação, definindo-se a forma de monitorização e mensuração do critério adotado (igualmente controlado pelo produtor), sendo possível detetar os desvios em tempo oportuno (devem estar disponíveis medidas preventivas) (Sorensen et al., 2004). Além disso, o próprio sistema de monitorização deve ser de natureza prática - o que parece ser de difícil aplicação às explorações pecuárias, pois nenhuma medida de controlo na exploração possuirá todas as características solicitadas por um PC (Cerf e Donnat, 2011).

Por exemplo, um tanque de leite pode permitir a monitorização do perigo e a definição de um limite crítico, mas a refrigeração não permite a prevenção da contaminação e não tem qualquer efeito na eliminação ou na redução do perigo. Alguns passos do processo não sendo quantificáveis, tornam difícil o estabelecimento de limites críticos e a adoção de um sistema de monitorização. Mesmo que haja esforços de monitorização para definir as necessidades de correção, as ações práticas apropriadas nem sempre são exequíveis.

Assim, a identificação de verdadeiros PC nas explorações parece ser problemático, mas possível de determinar em relação a perigos físicos e químicos (Cullor, 1997).

Para os potenciais perigos biológicos, os PC não são bem conhecidos e os limites críticos serão dificilmente definidos e aplicáveis em muitas das etapas de produção, podendo ser identificados até certo ponto recorrendo a metodologias epidemiológicas que quantifiquem os 
fatores de risco, em relação a determinadas doenças multifatoriais, que ocorrem ou podem ocorrer em diferentes áreas da exploração e que contribuem para a sua incidência e prevalência na exploração (Malher e Noordhuizen, 2008).

Estas metodologias contribuem para a identificação da fonte de infeção, mas irão diferir de exploração para exploração, uma vez que as condições e a estrutura variam com a exploração (Noordhuizen e Frankena, 1999). Provavelmente, os PC para muitos dos agentes patogénicos estarão associados às camas, à água, aos resíduos (águas residuais, chorume e estrume), aos locais tais como a enfermaria, as maternidades, o tanque do leite, e ainda os animais jovens, os adquiridos e os de refugo.

De qualquer forma, será necessário um sistema com poucos $\mathrm{PC}$. Nem todos os parâmetros serão controlados pelos PC, outros serão pelos pré-requisitos.

Face às dificuldades referidas, surgiu o conceito de POPA (Points of particular attention) (Malher e Noordhuizen, 2008), referenciados noutros casos como CMP - Critical Management Point (Lievaart et al., 2005). Enquanto os PC deverão obedecer a todos os critérios definidos pelo HACCP, os POPA falham um ou mais critérios.

A adoção do conceito de POPA prende-se com muitas das questões, relacionadas com doença nos animais, que mostram uma variação biológica (por exemplo a distribuição da frequência dos títulos serológicos $\rightarrow$ definição do limiar de positividade) e pela dificuldade na definição de normas estritas e tolerâncias / limites para títulos sorológicos.

Os POPA têm normalmente valores alvo a atingir numa exploração em particular e não valores padronizados com limites de tolerância, monitorizados por observação, mensuração ou testagem. A frequência de monitorização pode ser diária, semanal, mensal, anual.

A árvore de decisão utilizada na indústria alimentar tem sido considerada não adequada à produção animal pois algumas questões são difíceis de interpretar quando aplicadas a animais e plantas, pelo que têm sido desenvolvidas outras árvores de decisão no sentido de se ultrapassarem as dificuldades encontradas, e estruturadas como complemento à árvore de decisão do sector industrial alimentar (ILSI Europe, 1998; Department of Health and Ageing, Australian Government, 2007; Noordhuizen et al., 2008; Silva e Noordhuizen, 2008; Horchner e Pointon, 2011).

Quando um perigo permanece e não existindo um PC, a ISO 22000 recomenda o estabelecimento de um programa de pré-requisitos operacionais (PRo). O PRo é essencial para controlar a probabilidade de introdução do perigo alimentar e / ou a contaminação ou proliferação de perigos para a segurança alimentar no produto ou no ambiente de processamento.

Em relação aos limites críticos, ao nível da União Europeia, o Regulamento (CE) 853/2004 inclui regras de higiene para os produtos de origem animal, sendo requerida a amostragem de acordo com critérios específicos, nomeadamente em função dos critérios microbiológicos (Regulamento (CE) 2073/2005) e os níveis de resíduos em antibióticos (Regulamento 37/2005).

(CE)

Outra legislação introduz limites críticos em relação aos perigos químicos, como as micotoxinas (Regulamento (CE) 1881/2006), mas cuja aplicação à produção primária nem sempre poderá ser exequível, pelo menos por rotina. 
Alguns dos agentes zoonóticos como Brucella melitensis, Brucella bovis e Mycobacterium spp. são alvo de legislação específica e objeto de programas de erradicação cofinanciados pela UE, onde são definidos limites críticos objetivos, como por exemplo a impossibilidade de produzir queijo a partir de leite cru em rebanhos infetados por Brucella spp.

Realisticamente, deve-se considerar que face aos objetivos de segurança alimentar, os limites críticos não têm necessariamente de ser iguais aos definidos para o consumidor, podendo ser definidos objetivos e critérios de performance (Meyer-Broseta e Evain, 2004; ICMSF, 2005; Gorris, 2005), permitindo às explorações pecuárias selecionar as medidas apropriadas para atingir um determinado nível de segurança desejado e possível na produção primária, de acordo com os objetivos de segurança alimentar pretendidos, dependendo no entanto do produto final ser vendido diretamente ao consumidor na exploração pecuária ou a outro interveniente da fileira.

\section{Passo 10 (Princípio 5) - Medidas corretivas}

Este passo visa estabelecer as medidas corretivas a ser tomadas, quando a monitorização indicar que um PC não está sob controlo.

Em relação aos riscos biológicos, a Diretiva 2003/99/CE definiu os requerimentos necessários para a implementação de um sistema de monitorização e notificação anual de dados respeitantes às zoonoses, aos agentes zoonóticos, à resistência microbiana e a surtos de toxinfeções alimentares, dados que são analisados pela EFSA (European Food Safety Authority). Os dados respeitantes aos casos humanos são coligidos pela ECDC (European Centre for Disease Prevention and Control), permitindo um fluxo de informação permanente e à avaliação de riscos biológicos (Berthe et al., 2013).

Há medidas que podem controlar mais de que um perigo, mas outros perigos só são controlados com várias medidas, pelo que se torna necessário definir um plano de ação.

Os perigos biológicos podem ser reduzidos através de medidas de prevenção, inseridas nos planos de erradicação ou em programas de biossegurança, ou pela adoção e implementação de boas práticas de gestão da saúde animal com especial atenção para os agentes zoonóticos ou com impacto na qualidade.

Pode ainda recorrer-se a etapas subsequentes que possam contribuir para eliminar ou reduzir o perigo a um nível aceitável (árvore de decisão), como seja a pasteurização. Mas a sua eficácia dependerá das práticas introduzidas nas explorações que ajudem a mitigar os níveis bacterianos presentes (Maunsell e Bolton, 2004). Importa referir que a transferência de um perigo da exploração pecuária para a indústria significa necessariamente um impacto económico para esta última.

As características-chave para a erradicação de um agente patogénico implicam a existência de um único hospedeiro, a não existência de um reservatório selvagem, a produção de uma doença clínica identificável e a existência de um meio de diagnóstico eficiente (Cullor, 1997). Estas características não são facilmente encontradas, pelo que o objetivo passa pela redução do risco a um nível aceitável.

Devem ser providenciados mecanismos de monitorização e de recolha de informação necessária, para a realização de ações corretivas. No caso das doenças objeto de programas de erradicação, estes mecanismos estão criados, como já referido.

A implementação de um regime de testagem pode permitir determinar a 
tolerância ou limites críticos em relação a agentes infeciosos, a medicamentos de uso veterinário e a resíduos químicos nos alimentos de origem animal. No entanto, há um custo económico que deve ser previamente avaliado, face ao elevado número de perigos que podem ser encontrados numa exploração.

Ambas as medidas preventivas e corretivas contribuem para a redução ou eliminação do risco, mas é importante atender ao facto de que os animais vivos mostram uma considerável variabilidade biológica, pelo que a recuperação do controlo do processo, uma vez perdido, pode não ser garantido através de medidas de gestão de risco na exploração, embora contribuam para a redução do risco.

Para alguns fatores de risco tais como a presença de animais selvagens, incluindo as aves, é difícil considerar mesmo uma redução de risco, dificultando a definição de região indemne de uma doença que afete igualmente estes animais. Outros agentes, como aqueles que se podem dispersar pelo vento, são de difícil controlo.

Assim, este passo obriga a um empenhamento e dedicação por parte da equipa de HACCP, pois 0 conhecimento da exploração como um todo e a abordagem holística (Figura 2) poderão ajudar a contornar as situações mais complicadas.
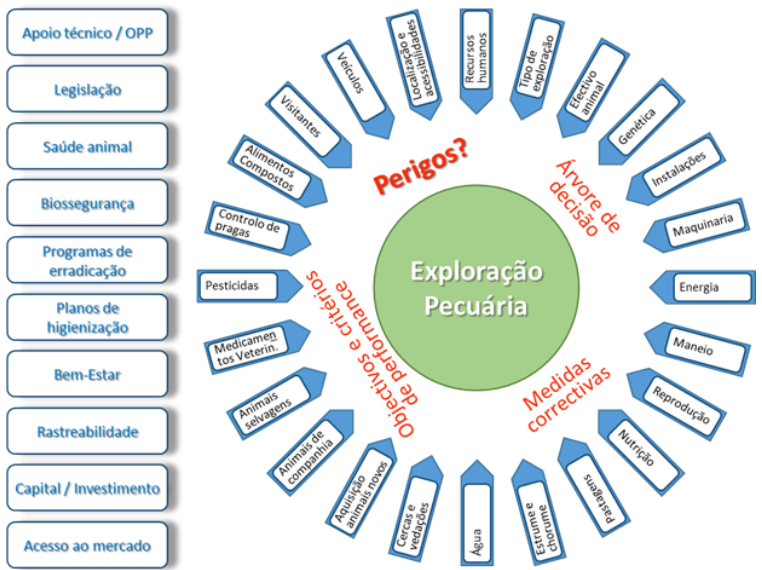

Figura 2 - Abordagem holística a uma exploração pecuária.
Passo 11 (Princípio 6)

O objetivo desta etapa é determinar os procedimentos de verificação e de validação interna e externa, que podem incluir testes suplementares e procedimentos que confirmem a operacionalidade do sistema HACCP, dando garantia ao consumidor de que o produto em questão é seguro e produzido de acordo com determinados procedimentos de produção documentados, que permitirá a sua certificação.

Se a verificação for realizada por agências independentes que estão acreditadas por organizações a nível nacional ou internacional, poderemos falar de Certificação, nomeadamente pela ISO 9000.

Para além da verificação é necessário validar o processo para se obter a evidência de que, a medida de controlo ou uma combinação de medidas bem implementadas, é capaz de controlar o perigo.

A validação é realizada ou no momento de aplicação da medida de controlo ou quando o sistema de controlo da segurança é delineado, ou ainda quando as alterações indicam a necessidade de revalidação.

O processo de validação é hoje, considerado como parte do contrato de compra pelas cadeias de distribuição, nomeadamente no que se refere aos registos de saúde animal dos efetivos e das práticas de produção recorrendo, por exemplo, a um esquema longitudinal integrado de garantia de segurança (LISA - Longitudinal Integrated Safety assurance) (Collins e Hall, 2004).

\section{Passo 12 (Princípio 7)}

Com esta etapa pretende-se estabelecer a documentação de todos os procedimentos e registos apropriados a estes princípios e às suas aplicações, que permitam a demonstração a uma terceira entidade, face à desejada certificação e como componente do processo de rastreabilidade. 
Esta documentação garante a transparência do processo, consagrada pelos acordos SPS e é um elemento chave na comunicação de risco (Snijders e Van Knapen, 2002).

\section{CONCLUSÃO}

Os produtores pecuários estão fundamentalmente preocupados com a saúde dos seus animais e como isso afeta os parâmetros de produção e a rentabilidade da exploração e menos interessados nos aspetos de saúde pública (Berman e Shimshony, 2013). Daí que, para além das doenças abrangidas pelos programas de erradicação, a sua preocupação se reduza maioritariamente a problemas de saúde do efetivo, que incluem alguns de carácter não infecioso. No entanto, a questão do valor pago pelo produto final é uma preocupação dos produtores, devendo estes repensar a forma de valorização desse produto, encontrando mais-valias que os satisfaçam.

A competitividade da produção de alimentos está cada vez mais dependente da confiança na segurança e qualidade e da aceitação dos procedimentos de produção, podendo ser aplicados a três níveis: produto, processo ou sistema.

Assim, os mercados estão cada vez mais orientados para a qualidade, com uma grande influência do consumidor sobre a produção animal (Noordhuizen e Frankena, 1999; Blaha, 2001) e vistos pela União Europeia como o principal princípio orientador para o mercado e para a proteção do consumidor (Blaha, 1999; Noordhuizen e Silva, 2007) e não para a quantidade e preço da produção de alimentos.

A definição de qualidade deixou de estar associada apenas ao produto, sendo estendida ao processo de produção, pelo que a saúde animal, o seu bem-estar, o ambiente de produção, a segurança dos alimentos e a saúde pública se tornaram questões principais, assim como a perceção do consumidor sobre estas questões (Noordhuizen e Metz, 2005).

A qualidade dos produtos traduzse não só pelo respeito das suas características intrínsecas, mas pelas medidas que asseguram a segurança dos mesmos, satisfazendo as necessidades do produtor e dos consumidores. Por isso, aumenta a importância, em relação à eficiência de produção de produtos de qualidade reconhecida pelo mercado.

Isso só é conseguido pela aplicação de sistemas de garantia da qualidade ao longo das cadeias de produção, incluindo a produção primária, tendo em conta que uma avaliação do produto no final da cadeia da sua produção não permite a correção de eventuais falhas ou melhorias, podendo inclusive quebrar a confiança dos consumidores.

Os sistemas de garantia da qualidade permitem implementar procedimentos de avaliação e verificação da qualidade e segurança dos produtos, em todos os passos da sua produção, e introduzir de imediato medidas de correção.

Assim, o principal objetivo de um sistema de gestão da qualidade e segurança dos produtos alimentares é a de oferecer ao consumidor o que ele procura, e de encontrar os métodos apropriados para certificar esses produtos (Nardone, 2002). No entanto, o processo é complexo face à diversidade de instituições envolvidas no comércio dos produtos de origem animal.

Os sistemas de gestão de risco sanitário de natureza geográfica têm igualmente constituído uma limitação para o comércio internacional dos produtos e mercadorias de origem animal e ao animal vivo, principalmente devido aos perigos biológicos, nomeadamente os responsáveis pelas doenças animais transfronteiriças 
(TADs), devendo-se ter em conta o contexto em que a doença circula (cadeia de valor) e os regulamentos existentes (ambiente institucional).

Importa referir que estes perigos biológicos circulam ao longo de toda a cadeia de valor em ambas as direções (da exploração ao consumidor), podendo ser mantidos em qualquer ponto da cadeia ou ser introduzida por fontes externas. A OIE não recomenda formalmente o HACCP como um sistema para a gestão de risco da doença animal nas explorações pecuárias, recomendando a adoção do CBT (Commodity-Based Trade), mas tem sido referenciada a inexistência de padrões internacionais, não havendo mecanismos de acreditação para muitas das doenças animais.

Através da compartimentação, deve ser alcançado um acordo bilateral entre os parceiros comerciais, de preferência com base em diretrizes e normas da OIE. Tem sido apontado uma abordagem integrada da cadeia de valor para a gestão do risco sanitário, nomeadamente com uma combinação do HACCP com o CBT (Thomson, 2014, Thomson et al., 2013).

Em Portugal, tal como noutros países da UE, o sistema de HACCP não tem sido aplicado de forma sistemática. Muitas explorações têm optado pelo GlobalGap (padrões definidos pelo sector privado), como forma de obterem mais-valias num mercado globalizado. $A$ nível da produção de leite de vaca, a indústria tem liderado um processo de aplicação de boas práticas de higiene ao nível das explorações. Os programas de biossegurança têm tido maior desenvolvimento a nível das explorações de aves e suínos em produção intensiva. Em ambos os casos, estes programas são facilmente utilizados pelo sistema HACCP.

Em outras espécies animais, como os pequenos ruminantes, explorados num sistema extensivo e onde se produzem queijos a partir do leite cru, utilizando-se em alguns casos metodologias de produção tradicionais, urge incentivar os produtores e as suas organizações a empreender a introdução de boas práticas que conduzam a um sistema de garantia da segurança e da qualidade dos seus produtos, já que é conhecida a relutância de muitos países, sobretudo do norte da Europa, em relação a estes produtos.

O recurso a um sistema de garantia da segurança e qualidade deve envolver todos aqueles que desenvolvem a sua atividade na fileira, para que o mesmo possa ser explicado e entendido pelo produtor. Estes sistemas obrigam a um investimento que só com sucesso na sua aplicação, haverá um retorno económico. Aqui, os Médicos Veterinários têm um papel muito importante a desempenhar, sobretudo ao nível das explorações pecuárias.

\section{REFERÊNCIAS}

ADAM, K.; BRÜLISAUER, F. The application of food safety interventions in primary production of beef and lamb: A review. International Journal of Food Microbiology, v.141, p.43-52, 2010.

ALEXOUPOULOS, C.; KRITAS, S.K.; PANAGIOTIS, D.T. et al. On-farm veterinary management programme (VMP) for the production of enhanced hygienic quality animal origin food products, biosecurity measures and on farm HACCP-compatible systems. In: International Society for Animal Hygiene, 2004, Sant-Malo, France.

BAINES, R.N.; RYAN, P.J.; DAVIES, W.P. HACCP at farm level - the missing link in food safety and security. In: Annual IAMA Conference in Montreaux, $14^{\text {th }}, 2004$, Montreaux.

BEEKHUIS-GIBBON, L.; WHYTE, P.; O'GRADY, L. et al. A HACCP-based 
approach to mastitis control in dairy herds. Part 1: Development. Irish Veterinary Journal, v.64, n.2, p.e1-e9, $2011^{a}$

BEEKHUIS-GIBBON, L.; DEVITT, C.; WHYTE, $P$. et al. A HACCP-based approach to mastistis control in dairy herds. Part 2: Implementation and evaluation. Irish Veterinary Journal, v.64, n.7, p.e1-e9, 2011 ${ }^{\mathrm{b}}$.

BERTHE, F.; RUGAS, M.; MAKELA, P. Integrating surveillance of animal health, food pathogens and foodborne disease in the European Union. Revue scientifique et technique International Office of Epizootics, v.32 n.2, p.521528, 2013.

BLAHA, T.H. Epidemiology and quality assurance application to food safety. Preventive Veterinary Medicine, v.39, p.81-92, 1999.

BLAHA, T.H. [2001]. The importance of Quality assurance and Food Safety in Modern Food Production Systems. Research Consortium Animal Production. Disponível em: http://www.ussoymeal.org/ruminant_s.pd f. Acesso em: 12/02/2017.

BERMAN, E.M.; SHIMSHONY, A. Integrating animal health and foodborne disease surveillance. Revue scientifique et technique International Office of Epizootics, v.32, n.2, p.337346, 2013.

BOERSEMA, J.S.; NOORDHUIZEN, J.P.; VIEIRA, A.,et al. Imbedding HACCP principles in dairy herd health and production management. Irish Veterinary Journal, v.61, n.9, 594-602, 2008

BONDE, M.; SORENSEN, J.T. Herd health management in organic production using a quality assurance system based on Hazard analysis and Critical Control Points. Netherlands. Journal of Agriculture Sciences, v.52, p.133-143, 2004.
BRUGĖRE-PICOUX, J.; KODJO, A. Actualités sur les Zoonose Émergentes et Résurgentes - News on Emerging and Resurging Zoonoses. Bulletin de l'Académie Vétérinaire de France, v.160, n.4, 2007.

CARDOEN S.; VAN HUFFEL, $X$; BERKVENS, D. et al. Evidence-based semi-quantitative methodology for priorizing for food-borne zoonoses. Foodborne Pathogens and Diseases, v.6, p.1083-1096, 2009.

CERF, O.; DONNAT, E. Application of hazard analysis - Critical control points (HACCP) principles to primary production: What feasible and desirable? Food Control, v.22, p.18391843, 2011.

CODEX ALIMENTARIUS COMISSIONCAC. Hazard analysis and Critical Control Point (HACCP). Principles and Guidelines for its Application. Genève, Switzerland: Codex Alimentarius Commission, Food Agricultural Organization, World Health Organization, 1997.

CODEX

ALIMENTARIUS COMMISSION-CAC. Draft principles and Guidelines for the Conduct of Microbiological Risk Assessment. ALINORM 99/13 Genève, Switzerland: Codex Alimentarius Commission, Food Agricultural Organization, World Health Organization, 1998.

COLEMAN, M.E.; MARKS, H.M. Qualitative and quantitative risk assessment. Food Control, v.10, p.289297, 1999.

COLLINS, J.D.; HALL, P.G. Food safety and animal production systems: controlling zoonoses at farm level. Revue scientifique et technique International Office of Epizootics, v.23, n.2, p.685-700, 2004.

CULLOR, J.S. HACCP (Hazard Analysis Critical Control Points): Is it coming to 
the dairy? Journal of Dairy Science, v.80, p.3449-3452, 1997.

DEPARTMENT OF HEALTH \&
AGEING. GOVERNMENT. Business Sector Food Safety Risk Priority Classification Framework. Food Regulation Standing Committee, 2007.

Farmers European and AgriCooperatives - COPA-COGECA [2015]. EU Guide to Good Hygiene Pratice (GGHP) for the primary production of foodstuffs. Disponível em: www.copacogeca.eu. Acesso em: 04/04/2017.

FOOD AND
ORGANIZATION OF THE

FORMATO, G.; SMULDERS, F.J. Risk management in primary apicultural production. Part 1: a Hazard Critical Control Point approach to assuring the safety of unprocessed honey. Veterinary Quarterly, v.31, n.1, p.2947, 2011.

FORMATO, G.; ZILLI, R.; CONDOLESO, $R$. et al. Risk management in primary apicultural production. Part 2: bee health and disease prevention and associated best practices. Veterinary Quarterly, v.31, n.2, p.87-97, 2011.

FRAZZOLI, C.; MANTOVANI, A. Toxicants exposure as novel zoonosis; reflections on sustainable development, food safety and veterinary public health. Zoonosis and Public Health, v.57, n.78, p.136-142, 2009.

GARDNER, I.A. Testing to fulfill HACCP (Hazard Analysis Critical Control Points).
Requirements: Principles and Examples. Journal of Dairy Science, v.80, p.34533457, 1997.

GORRIS. L.G.M. [2002]. The Impact of Risk Analysis on Food Safety. Inauguration address as the new holder of the European Chair in Food Safety Microbiology at Wageningen University, Wageningen. Disponível em: http://www.wur.nl/oraties/gprris 3-102002.pdf(2002). Acesso em: 13/03/2017

GORRIS, L.G. Food safety objective: An integral part of food chain management. Food Control, v.16, p.801-808, 2005.

HORCHNER, P.M.; POINTON, A.M. HACCP-based program for on-farm food safety for pig production in Australia. Food Control, v.22, p.1674-1688, 2011.

HUMBLET, M.F.; VANDEPUTTE, S.; ALBERT, A. et al. [2012]. Multidisciplinary and evidence-based method for prioritizing diseases of foodproducing animals and zoonosis emerging infectious disease. Disponível em:

http://wwwnc.cdc.gov/eid/article/18/4/111151_article.htm. Acesso em: 02/02/2017.

INTERNATIONAL LIFE SCIENCES INSTITUTE EUROPE - ILSI. A simple guide to understanding and applying the hazard analysis critical control point concept. 2nd ed. Brussels Belgium: Europe Scientific Committee of Food Safety, 1998.

International Commission on Microbiological Specifications for Foods - ICMSF. A simplified guide to understanding and using Food Safety Objectives and Performance Objectives. ICMSF, 2005. Disponível em:

http://www.icmsf.org/pdf/FSO\%200jectiv es/GuiaSimplificadoEnglish.pdf

KAITAZOV, G.; STOYANCHEV, T. Modified Hazard Analysis. Basic programs of the modified HACCP for 
poultry industry. Bulgarian Journal of Agriculture Science, v.14, n.5, p.466469, 2008.

KEENER, L. [2001]. Why HACCP systems are prone to failure. State of the art. Food Safety Magazine. Disponível em:

www.zipcon.net/ foodsafe/soao=haccp. pdf. Acesso em: 13/04/2017.

KOMOROWSKI, E. New dairy hygiene legislation. International Journal of Dairy Technology, v.59, n.2, p.97-101, 2006.

LIEVAART, J.J.; NOORDHUIZEN, J.P.; VAN BEEK, E. et al. The Hazard analysis and Critical Control Point's (HACCP) concept as applied to some chemical, physical and microbiological contaminants of milk on dairy farms: A prototype. Veterinary Quarterly, v.27, n.1, p.21-29, 2005.

MALHER, $X . ; \quad N O O R D H U I Z E N, \quad J . P$. Applying the HACCP principles to selected hazard during goat kids rearing on milking goat farms in western France.

Revue de Médecine Vétérinaire, v.159, n.1, p.38-48, 2008.

$\begin{array}{lll}\text { MAUNSELL, } & \text { B.; } & \text { BOLTON, D.J. } \\ \text { Guidelines } & \text { for food safety }\end{array}$
Republic of Irland: The Food Safety Department. Teagasc - The National Food Center, 2004. 31 p.

MEYER-BROSETA, S.; EVAIN, L. Principes et lignes directrices pour la gestion des risques microbiologiques: état des discussions en cours au sein du Codex Alimentarius à propos des concepts de Niveau approprié de protection, d'objectif de sécurité sanitaire des aliments, d'Objectif de performance et de Critère de performance. Bulletin De I'Academie Veterinaire De France, v.157, n.3. p4952, 2004.

MOLLER, S.H. Management of health in mink. A HACCP plan for energy allowance during winter and gestation in order to control sticky kids. In: International Scientific Congress in Fur Animal Production, VIII ${ }^{\text {th }}, 2004$, SHertogenbosh, The Nederlands.

Proceedings S-Hertogenbosh: Scientifur Reviewed Articles, 2004. v.28, n.3, p.50- 57.

MURRAY, N.; MACDIARMID, S.; WOOLDRIDGE, $M$. et al. Introduction and qualitative risk analysis. In: World Organization for Animal Health - OIE. Handbook on Import Risk Analysis for Animals and Animal Products. Paris, France: OIE, 2004a, v.1, p. 1-59.

MURRAY, N.; MACDIARMID, S.; WOOLDRIDGE, $M$. et al. Quantitative Risk Assessment. In: World Organization for Animal Health - OIE. Handbook on Import Risk Analysis for Animals and Animal Products. Paris, France: OIE, 2004b, v.2, p. 1-126.

NARDONE, A. Evolution of livestock production and quality of animal products. In: Annual Meeting of the Brazilian Society of Animal Science, $39^{\text {th }}, \quad 2002$, Recife, Pernambuco. Proceedings Recife: Brazilian Society of Animal Science, 2002. p. 486-513.

NETTLES, L.; BUKENYA, J.O. [2005]. Willingness to adopt HACCP: Goat producers survey results. Internet Journal of Food Safety, v.6, p.11-16, $2005 . \quad$ Disponível em: www.foodhaccp.com/internetjournal/ijfsv 6-3.pdf. Acesso em: 13/04/2017

NOORDHUIZEN, J.P.; SILVA, C.J. Herd health management and quality risk control on large dairy farms. In: INTERNATIONAL CONGRESS IN ANIMAL HYGIENE, XIIIth, 2007. Tartu, Estónia. Proceedings: Tartu: International Society for Animal Hygiene, University of Life Sciences, 2007. p. 311.

NOORDHUIZEN, J.P.; SILVA, C.J. [2009]. Animal Hygiene and Animal Health in Dairy Cattle Operations. The 
Open Veterinary Science Journal, v.3, p.17-21, 2009. Disponível em: https://benthamopen.com/contents/pdf/T OVSJ/TOVSJ-3-17.pdf. Acesso em: $13 / 04 / 2017$

NOORDHUIZEN, J.P.; SILVA, C.J. HACCP - based quality management approach to udder health problems on dairy farms. Irish Veterinary Journal, v. 62, Supplement, p. 21-25, 2009.

NOORDHUIZEN, J.P.; FRANKENA, K. Epidemiology and quality assurance: applications at farm level. Preventive Veterinary Medicine, v.39, p.93-100, 1999.

NOORDHUIZEN, J.P.; SILVA C.J.; BOERSEMA, S-J. et al. Critical Control Points and Points of Particular Attention: their standards and tolerances or targets, their monitoring and corrective measures. In: Applying HACCP-based Quality Risk Management on Dairy Farms. The Nedherlands: Wageningen Academic Publishers, 2008. Cap.7, p.109-126.

NOORDHUIZEN, J.P.; METZ, J.H. Quality control on dairy farms with emphasis on public health, food safety, animal health and welfare. Livestock Production Science, v.94; p.51-59, 2005.

NOORDHUIZEN, J.P.; WELPELO, H.J. Sustainable improvement of animal of animal care by systematic risk management according to HACCP. Veterinary Quarterly, v.18, p.121-126, 1996.

PANISELLO, P.J.; QUANTICK, P.C. Technical barriers to Hazard Analysis Critical Control Point (HACCP). Food Control, v.12, p.165-173, 2001.

REGULAMENTO (CE) No 178/2002 DO PARLAMENTO EUROPEU E DO CONSELHO de 28 de Janeiro de 2002 que determina os princípios e normas gerais da legislação alimentar, cria a Autoridade Europeia para a Segurança dos Alimentos e estabelece procedimentos em matéria de segurança dos géneros alimentícios.

REGULAMENTO (CE) N. ${ }^{\circ} 852 / 2004$ DO PARLAMENTO EUROPEU E DO CONSELHO de 29 de Abril de 2004 relativo à higiene dos géneros alimentícios.

REGULAMENTO (CE) N. ${ }^{\circ} 853 / 2004$ DO PARLAMENTO EUROPEU E DO CONSELHO de 29 de Abril de 2004 que estabelece regras específicas de higiene aplicáveis aos géneros alimentícios de origem animal.

REGULAMENTO (CE) N. ${ }^{\circ}$ 854/2004 DO PARLAMENTO EUROPEU E DO CONSELHO de 29 de Abril de 2004 que estabelece regras específicas de organização dos controlos oficiais de produtos de origem animal destinados ao consumo humano

REGULAMENTO (CE) No 37/2005 DA COMISSÃO de 12 de Janeiro de 2005 relativo ao controlo das temperaturas nos meios de transporte e nas instalações de depósito e armazenagem de alimentos ultracongelados destinados à alimentação humana.

REGULAMENTO (CE) No 183/2005 DO PARLAMENTO EUROPEU E DO CONSELHO de 12 de Janeiro de 2005 que estabelece requisitos de higiene dos alimentos para animais.

REGULAMENTO (CE) No 2073/2005 DA COMISSÃO de 15 de Novembro de 2005 relativo a critérios microbiológicos aplicáveis aos géneros alimentícios.

REGULAMENTO (CE) No 1881/2006 DA COMISSÃO de 19 de Dezembro de 2006 que fixa os teores máximos de certos contaminantes presentes nos géneros alimentícios.

REGULAMENTO (UE) No 1306/2013 DO PARLAMENTO EUROPEU E DO CONSELHO de 17 de dezembro de 2013 relativo ao financiamento, à gestão e ao acompanhamento da Política 
Agrícola Comum e que revoga os Regulamentos (CEE) $n^{\circ} 352 / 78,(C E) n^{\circ}$ 165/94, (CE) $n^{\circ}$ 2799/98, (CE) $n^{\circ}$ 814/2000, (CE) no 1290/2005 e (CE) $n^{\circ}$ 485/2008 do Conselho.

REGULAMENTO (UE) No $1307 / 2013$ DO PARLAMENTO EUROPEU E DO CONSELHO de 17 de dezembro de 2013 que estabelece regras para os pagamentos diretos aos agricultores ao abrigo de regimes de apoio no âmbito da política agrícola comum e que revoga o Regulamento (CE) n.o 637/2008 do Conselho e o Regulamento (CE) $n^{\circ}$ 73/2009 do Conselho.

REGULAMENTO (UE) No 640/2014 DA COMISSÃO de 11 de março de 2014 que completa o Regulamento (UE) $n^{\circ}$ 1306/2013 do Parlamento Europeu e do Conselho no que diz respeito ao sistema integrado de gestão e de controlo e às condições de recusa ou retirada de pagamentos, bem como às sanções administrativas aplicáveis aos pagamentos diretos, ao apoio ao desenvolvimento rural e à condicionalidade.

REGULAMENTO (UE) No 907/2014 DA COMISSÃO de 11 de março de 2014 que completa o Regulamento (UE) $n^{\circ}$ 1306/2013 do Parlamento Europeu e do Conselho no que se refere aos organismos pagadores e outros organismos, à gestão financeira, ao apuramento das contas, às garantias e à utilização do euro).

ROMERO-BARRIOS, P.; HEMPEN, M.; MESSENS, W. et al. Quantitative microbiological risk assessment (QMRA) of food-borne zoonosis at European level. Food Control, v. 29: p.343-349, 2013.

ROSENBERG, F.J. Social structure and veterinary epidemiology in Latin America. Boletin del Centro Panamericano de Fiebre Aftosa, v. 52, p.25-45, 1986.
RUEGG, P.L. Practical Food Safety interventions for Dairy Production. Journal of Dairy Science, v.86: (E. Suppl.), p.1-9, 2002.

SILVA, J.C.; NOORDHUIZEN, J.P. Consumer safety and HACCP-like quality risk managements programs on Dairy Farms: the role of veterinarians. The Open Veterinary Science Journal, v.2, p. 37-49, 2008.

SNIJDERS, J.M.; VAN KNAPEN, F. Prevention of human diseases by an integrated quality control system. Livestock Production Science, v.76, p. 203-206, 2002.

SORENSEN, J.T.; ROUSING, T.; KLAAS, I.C. Surveillance and management in dairy cattle after implementation of automatic milking: is the HAACP concept useful? In: CONGRESS OF THE INTERNATIONAL SOCIETY FOR ANIMAL HYGIENE: ANIMAL PRODUCTION IN EUROPE: THE WAY FORWARD IN A CHANGING WORLD "IN-BETWEEN", 2004, Saint Malo, France. Proceedings: Saint Malo: International Society for Animal Hygiene, 2004. p.499-500.

SORENSEN, J.T.; BLOND, M.; ROUSING, $T$. et al. Herd health surveillance and management in an integrated HACCP based system. In: SYMPOSIUM OF THE INTERNATIONAL SOCIETY FOR VETERINARY EPIDEMIOLOGY AND ECONOMICS, $11^{\text {th }}$, 2006, Cairns, Australia. Proceedings: Cairns: International Society for Veterinary Epidemiology And Economics, 2006. p.1-4.

SOSSIDOU E.N.; RAMANANTIS, S.B.; MANTIS, F.N. et al. On farm welfare risks influencing the quality of the traditional Mediterranean animal products. National Agricultural Research Foundation, Animal Research Institute, p.1-5, 2015. 
SPERBER, W.H. HACCP does not work from farm to table. Food Control, v.16, p.511-514, 2005.

TAYLOR, L.H.; LATHAM, S.M.; WOOLHOUSE, M.E. Risk factors for human disease emergence. Biological Sciences, Bristol, v. 356, p. 983-989, 2001.

THOMSON, G.R.; PENRITH, M-L.; ATKINSON, M.W. et al. International Trade Standards for Commodities and Products Derived from Animals: The need for a System that Integrates Food Safety and Animal Disease Risk Management. Transboundary and Emerging Diseases, v.60, n. 6, p. 507515, 2013.

THOMSON, G. Equivalence in relation to animal health risk management. In: SADC ANIMAL HEALTH TRAINING WORKSHOP, 2014, Gaborone, Botswana. Proceedings Botswana: Southern African Development Community, 2014. p. 29-31.

VAN DER SPIEGEL, M.; STERRENBURG, P.; HAASNOOT, W. et al. Towards a decision support system for control of multiple food safety hazard in raw milk production. Trends in Food Science \& Technology, v.34, p.137-145, 2013.

VILAR, M.J.; RODRIGUEZ-OTERO, J.L.; SANJUÁN, M.L. et al. Implementation of HACCP to control the influence of milking equipment and cooling tank on the milk quality. Trends in Food Science and Technology, v.23, p.4-12, 2012.

VOSE D. Terminologie en analyse de risqué. Épidémiologie et Santé Animal, v 41, p1-3, 2002.

VOSE, D. Risk analysis: a quantitative guide. England: Wiley, 2008. 752 p.

WOAH. Guide to good farming practice for animal production food safety. Scientific and Technical Review, v.25, n.2, p.823-836, 2006.
World Trade Organization - WTO [1995]. Agreement on the application of sanitary and phytosanitary measures. Genève, WTO, 1995. Disponível em: www.wto.org/english/tratop_e/sps_e/spr agr_e.htm. 